\title{
A Survey on collusion resistant data sharing in cloud environment
}

\author{
N.Rajkumar ${ }^{1} *$, E Kannan ${ }^{2}$ \\ ${ }^{1}$ Associate Professor , ${ }^{2}$ Professor \\ Department of Computer Science and Engineering, School of Computing \\ Vel Tech Rangarajan Dr. Sagunthala R\&D Institute of Science and Technology, Avadi, Chennai-62, TamilNadu \\ *Corresponding author E-mail: sivarajkumar.n@gmail.com
}

\begin{abstract}
Cloud Computing is an emerging technology that uses the internet and remote servers to save information and application. Cloud computing enables customers and organizations to utilize applications without establishment and access their own documents at any computer with internet access. Collusion Resistant Secured the way to deal the fulfillment of an abnormal state of security with complete protection. A large portion of these techniques depend on a presumption that semi-trusted and arrangement is absent. In this paper, we concentrate on the issue of conspiracies, in which a few gatherings may connive and share their record to find the private data of different gatherings. The other related works better than this is more secure. This paper reveals an overview and study of collusion resistance techniques in more secure and efficient way for data sharing in cloud storage.
\end{abstract}

\section{Introduction}

Cloud storage is a service model in which information is kept up, overseen, moved down remotely and made accessible to clients over a network (typically the Internet). Users for the most part pay for their cloud data storage on every utilization, monthly rate. Although the per-gigabyte cost has been profoundly determined down, cloud storage providers have added operating expenses that can make the innovation more costly than users anticipated. Cloud security keeps on being a worry among users. Providers have endeavored to manage those apprehensions by building security, abilities, for example, encryption and confirmation, into their services. Cloud storage is a model of data storage in which the digital data is stored in logical pools, the physical storage spans multiple servers (and often locations), and the physical environment is typically owned and managed by a facilitating company. These cloud storage providers are in charge of keeping the information accessible and available, and the physical environment protected and running. People and organizations buy or lease storage capacity from the providers to store user, organization, or application data. A type of security attack or threat in which a node intentionally makes a secret agreement with an adversary, or the node is somehow made to have such an agreement. In multiuser cloud computing there may be a major problem to securely share documents. Frequent change of membership, challenging issues to prevent the system from collusion attack, to secure the system from the revoked user. Agreement protection, as far as SS, implies keeping the qualities produced at any individual hub mystery, with the end goal that if different hubs share data together or plot, the mystery esteems can't be resolved. It is a testing issue to safely oppose the intrigue of cloud server and question clients while executing closest neighbor inquiry over encoded information in cloud. conspiracy protection includes expanding the quantity of individuals (or hubs) required to register the private information estimations of the rest of the people or hubs. Concentrate on the issue of intrigues, in which a few gatherings may conspire and share their record to find the private data of different gatherings. Plot insurance is then accomplished when no hub has the same neighboring hubs twice for each cycle. This perception is done in an arrangement safe PPDM calculation verifiably in view of two edge disjoint Hamiltonian cycles Another exceptional technique to giving intrigue protection by making shares yet without the requirement for edge-disjoint Hamiltonian cycles. a calculation for mining information that is impervious to arrangement by partaking destinations.

\section{Literature Review}

Samuel Shepard et al [1] exhibit his Privacy protecting Data mining (PPDM) seeks to enable clients to share data while guaranteeing individual and corporate security concerns are tackled. Numerous algorithms have been acquainted with keep up security notwithstanding when everything except two parties colludes. One intriguing bit of research depend more on the obscurity of qualities to accomplish a measure of security, that is, if a data miner can't make sense of which esteem has a place with whom, a level of protection is in this way managed when joined with cryptographic strategies. Instruments for protection saving circulated information mining present various valuable algorithmic natives for PPDM, including secure set association, secure size of convergence, and secure sum (SS). The objective of SS is basic: given the estimation of each site's individual a chance to include be covered while the worldwide entirety of all data sources is generally known. In other words, the individual site's security is saved. In this paper he depicted an algorithm for mining data that is impervious to conspire by taking part destina- 
tions. He additionally gave a numerical confirmation of the algorithm. Receptacle Yang et al [2] concentrate on the issue of arrangements, in which a few gatherings may intrigue and offer their record to conclude the private data of different gatherings. Specifically, he considers a general issue in PPDM - multiparty secure calculation of a few elements of secure summations of information spreading around different gatherings. To take care of such an issue, he proposes another strategy that involves an abnormal state of security - full-protection. With this technique, no delicate data of a gathering will be uncovered notwithstanding when every other gathering conspire. What's more, this technique is productive with a running time of $\mathrm{O}(\mathrm{m})$ and furthermore applying this general strategy, a substantial number of issues in PPDM can be settled with improved security. $\mathrm{He}$ summed up an extensive number of issues in protection safeguarding information mining into a formal issue, SPoS. He proposed a convention to safely process the estimation of that item. His proposed convention fulfills an abnormal state of security full-protection. It in this manner is more secure than other related techniques. His convention is additionally a proficient one since its running time is corresponding to the quantity of gatherings. Additionally, its proficiency can be enhanced further on the off chance that he diminishes the level of security. Likewise, a protected correspondence channel is superfluous for his convention in view of its high security. Since the protected calculation of capacities on secure summations is a general issue in protection safeguarding information mining, he proposed convention can be connected to take care of a large number of the issues in this field. Wei Yang et al [3] concentrated on protection saving Data Aggregation Schemes display another sort of agreement assault procedure called Hamburger Attack. It is of intelligent straight forwardness; however has the upside of being compelling and proficient. To utilize it to check the security of a few existing protection saving information collection plans. To demonstrate that under ground sirloin sandwich assault, some earlier security safeguarding information accumulation conventions will unveil part, even all, of the private information that they planned to ensure. Then again, the burger assault is advantageous to outlining new protection safeguarding information total plans. It can help them in staying away from this sort of conspiracy assault show another kind of agreement assault which is known as a Hamburger Attack. The objective is to both discover and defeated the security provisos that existed in some past protection safeguarding information total plans utilizing this assault procedure. The name Hamburger Attack originates from its three-layer structure: A thick cut ("bread") at the main, a thin cut ("meat") in the center, and a thick cut ("bread")at the base. The best and base layers are thought to be foes or debased members who are joined together to dispatch an assault. The center layer should be the casualty whose private information is in question.

Hamburger Attack

In protection saving information total plans, a standout amongst the most habitually considered inquiries is to what degree a conglomeration plan can oppose arrangement assault of members. On the off chance that a motivation good accumulation convention can endure up to $\mathrm{k}$ ruined members whose activities are straightforward yet inquisitive, and say that this convention is $\mathrm{k}$ safe. Clearly, the extraordinary instance of 1-safe is inconsequential, for this situation the collection convention is basically not ready to oppose any plot assault. Along these lines, the number $\mathrm{k}$ is constantly equivalent to or more noteworthy than 2 by and by. In our Hamburger Attack demonstrate, it just needs two members to be adulterated. This is the base number of plotting parties, along these lines it requires minimal assets to dispatch a conspiracy assault. In other words, if a security protecting information conglomeration convention is demonstrated uncertain under Hamburger Attack, it doesn't accomplish even the base 2-safe.
Renren Dong et al [4] concentrated on Multiparty Computation utilizes another confide in display for arrange PCs. At that point utilize this model as a premise to enhance the intrigue protection ability of information mining calculations. To utilize an execution metric to evaluate the change. Numerous Secure multiparty calculation (SMC) strategies have been created to explain various subordinates of SMC issues Some of these techniques depend on the utilization of Hamiltonian cycles(HCs). In trust show call a SMC as HC-based, otherwise called HSMC, if the calculation of the calculation depends on at least one HCs to get the worldwide outcome. HCs assumes an essential part in diagram hypothesis and information mining applications. Hubs partake in the mining calculation by trading messages along a HC. The objective is to process a worldwide mining amount in a disseminated way without anybody knowing who contributed an incentive to the worldwide calculation. By having numerous cycles to pass messages around, the private esteem can be kept mystery regardless of whether hubs take part in untrustworthy conduct - for instance a gathering of hubs plot to find another hub's private esteem. To start with utilizes a model for trust and characterize a metric for estimating the security of a given topology. At that point indicate how the security factor can be enhanced by utilizing a friendlier way, or an altered cycle, for the mining. Dipali S. Kasunde et al [5] Narrated Multi-Owner Shared Data will give open evaluating on multi proprietor shared information. At the point when client is renounced from the gathering, there must be some technique to leave those hinders that are marked by that denied client. Multi-Owner Shared Data will likewise give productive client denial conspiracy protection i.e. regardless of whether cloud plots with any denied clients; it won't comprehend the substance of the information which is put away on cloud. Multi-Owner Shared Data will comprise of the three principle elements gathering of various clients, open verifier and the cloud. Gathering comprises of various clients which will impart the information to one another; they can be multi-proprietor of the information. It comprises ofmanager of a gathering which can produce security parameters and to deny clients. Remained clients in the gathering are the enrolled clients that won't just store their own information into the cloud yet in addition share them with each other. Publicverifier can be a TPA (outsider reviewer) generally customer which uses the common information for particular reason which gives check benefit on the honesty of information, utilizing test and reaction convention with clients. At the point when client will be repudiated from the gathering, there ought to be re-age of marks on pieces of information which are signedby the disavowed client. This module will utilize the possibility of intermediary multi-signature. The intermediary underwriter will take open keys of the considerable number of clients (aside from denied client) and re-figure theproxy signature for every unique proprietor. Framework will ensure the conspiracy assault i.e. cloud won't get the first information document regardless of whether they trade off with the renounced client. At the point when client will be repudiated from the gathering, bunch chief will expel this client from the rundown and refresh the information documents to the cloud encoded with new re-encryption key. As the disavowed client couldn't recuperate re-encryption key cloud won't comprehend the substance of the information document put away on cloud. Regardless of whether they will recuperate or plot it negates with the Discrete Logarithm (DL) presumption and Computational Diffie Hellman Assumption (CDH) and. So the framework willbe plot safe. The intermediary multisignature plan to be used in this Multi-Owner Shared Data can be useful for various associations having number of offices, for example, budgetary, designing, and so forth to check trustworthiness of multi proprietor archive. You wen Zhu et al [6] portrays Collusion-Resisting Secure Nearest Neighbor Query gives a productive assault technique which shows CloudBI-II will uncover the distinction vectors under the conspiracy assault. Further, to demonstrate that the distinction vector exposure will 
bring about genuine security rupture, and consequently achieve a proficient assault technique to break CloudBI-II. In particular, CloudBI-II cannotachieve their pronounced security. This assault approach can quick recuperate the first information from the encoded informational collection in Cloud BI-II. At long last, give an improved plan which can productively oppose the intrigue assault. In this work approaches a productive assault strategy which indicates CloudBI-II in isn't secure. In this assault, first indicated CloudBI-II will reveal the distinction vectors of DO's database focuses, and afterward demonstrated the distinction vector exposure will prompt genuine security rupture. Hypothetical investigation and examination assessment showed our assault approach is of high effectiveness. Furthermore, we displayed an improved plan to safely oppose the plot assault.

Larry A. et al [7] states Anonymous ID Assignment for mysterious sharing of private information among $\mathrm{N}$ parties is created. This method is utilized iteratively to allocate these hubs ID numbers extending from 1 to $\mathrm{N}$. This task is mysterious in that the personalities got are obscure to alternate individuals from the gathering. Protection from plot among different individuals is confirmed in a data theoretic sense when private correspondence channels are utilized. This task of serial numbers enables more unpredictable information to be shared and has applications to different issues in security safeguarding information mining, crash evasion in interchanges and dispersed database get to. The required calculations are dispersed without utilizing a confided in focal expert. New calculations for allocating mysterious IDs are inspected concerning exchange offs amongst correspondence and computational necessities. This work manages effective calculations for appointing identifiers(IDs) to the hubs of a system such that the IDs are unknown utilizing an appropriated calculation with no focal expert. Given hubs, this task is basically a change of the whole numbers with every ID being known just to the hub to which it is alloted. The principle calculation depends on a technique for namelessly sharing straightforward information and results in strategies for proficient sharing of complex information. There are numerous applications that require dynamic one of a kind IDs for arrange hubs. Such IDs can be utilized as a feature of plans for sharing/separating correspondences transmission capacity, information stockpiling, and different assets secretly and without struggle. Utilization of the Newton characters extraordinarily diminishes correspondence overhead. This can empower the utilization of a bigger number of "openings" with a subsequent diminishment in the quantity of rounds required. The arrangement of a polynomial can be maintained a strategic distance from to some detriment by utilizing Sturm's hypothesis. The advancement of an outcome like the Sturm's strategy over a limited field is an alluring probability with private correspondence channels, our calculations are securein a data theoretic sense. Obviously, this property is exceptionally delicate. The fundamentally the same as issue of mental poker was appeared to have no such arrangement with two players and three cards. The contention of can without much of a stretch be reached out to, e.g., two sets every one of plotting players with a deck of cards instead of our deck of cards. As opposed to limits on fruition time created in past works, our formulae give the normal fulfillment time precisely. To guess the asymptotic equation of Corollary, in view of computational experience, to be a genuine upper bound.

\section{Conclusion}

In this paper we presented the survey for issues of data sharing in cloud storage by collusion. The vast majority of these techniques depend on a suspicion that semi-legitimate and conspiracy is absent. We concentrate on the issue of conspiracies, in which a few users may collude and share their record to conclude the private data of different users. This work demonstrates that there are a few techniques to enhance the collusion with various methodologies. Different collusion techniques are surveyed which enhance the current algorithm with alternate point of view.

\section{References}

[1] Samuel Shepard+, Ray Kresman*, Larry Dunning* .Data Mining and Collusion Resistance, Proceedings of the World Congress on Engineering 2009 Vol I WCE 2009, July 1 - 3, 2009, London, U.K.

[2] Bin Yang Collusion-Resistant Privacy-Preserving Data Mining,Bin YangGraduate School of Information Science and Technology University of Tokyo, Japan yangbin@r.dl.itc.utokyo.ac.jp

[3] Wei Yang1,2,*, Liusheng Huang1 Hamburger Attack: A Collusion Attack againstPrivacy-preserving Data Aggregation Schemes. 2015 IEEE 23rd International Symposium on Quality of Service (IWQoS)

[4] Renren Dong. Trust Enabled Secure Multiparty Computation. 2010 14th International Conference Information Visualisation

[5] Dipali S. Kasunde. Verification of Multi-Owner Shared Data with Collusion Resistant User Revocation in Cloud, 2016 International Conference on Computational Techniques in Information and Communication Technologies (ICCTICT).

[6] You wen Zhu, Collusion-Resisting Secure Nearest Neighbor Query over Encrypted Data in Cloud, Revisited, College of Computer, Nanjing University of Aeronautics and Astronautics, Nanjing, China

[7] Larry A. Privacy Preserving Data Sharing With Anonymous ID Assignment, ieee transactions on information forensics and security, vol. 8 , no. 2 , february 2013

[8] D. Beaver, S. Micali, and P. Rogaway, "The round complexity of secure protocols," in STOC. ACM, 1990.

[9] A. Ben-David, N. Nisan, and B. Pinkas, "Fairplaymp: a system for secure multi-party computation," in CCS. ACM, 2008.

[10] D. Boneh, "The decision diffie-hellman problem," Algorithmic Number Theory, 1998 